\title{
A LOW-COST NON-INTERCEPTING BEAM CURRENT AND PHASE MONITOR FOR HEAVY IONS*
}

\author{
J. M. Bogaty and B. E. Clifft \\ Physics Division, Argonne National Laboratory, Argonne, IL 60439
}

\section{INTRODUCTION}

A low cost ion beam measurement system has been developed for use at ATLAS [1,2]. The system provides nondestructive phase and intensity measurement of passing ion beam bunches by sensing their electric fields. Bunches traverse a short tubular electrode thereby inducing displacement currents. These currents are brought outside the vacuum jacket where a lumped inductance resonates electrode capacitance at one of the bunching harmonic frequencies. This configuration yields a basic sensitivity of a few hundred millivolts signal per microampere of beam current. Beam induced radiofrequency signals are summed against an offset frequency generated by the master oscillator. The resulting difference frequency conveys beam intensity and bunch phase information which is sent to separate processing channels. One channel utilizes a phase locked loop to stabilize phase readings during microsecond beam drop outs. The other channel uses a linear full-wave active rectifier circuit which converts sine wave signal amplitude to a D.C. voltage representing beam current. Plans are in progress to install this new diagnostic at several locations in ATLAS which should help shorten the tuning cycle of new ion species.

\section{BEAM DETECTION}

Figure 1 shows a schematic representation of the prototype beam measurement system in use at ATLAS. The beam pickup electrode design features an external resonant $R F$ tank circuit which can be changed to tune any one of several bunching harmonics. Since the tubular pickup electrode is the only element under vacuum, cost of construction is lower than spiral or helical RF cavities. There is a trade off in beam sensitivity compared to RF cavity designs $[3,4]$ but this is partially offset by using phase locked loops and active bandpass filters to minimize random noise effects. Fourier analysis of bunched beam passing through the pickup electrode was used to determine a general expression for signal amplitude across inductor $\mathrm{L}_{\mathrm{T}}$ :

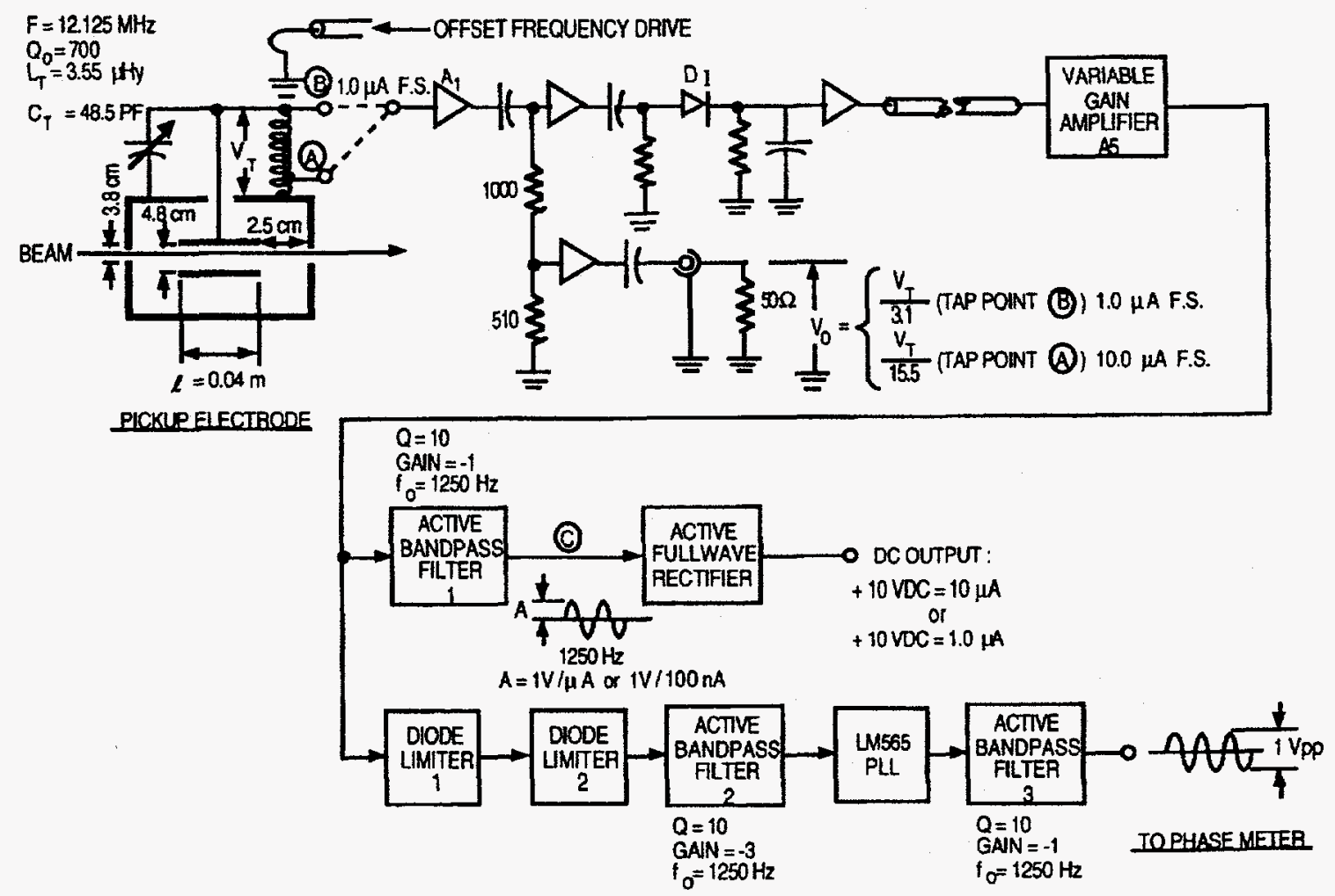

Fig. 1

The submitted manuscript has been authored by a contractsr of the U.S. Government by a contractsr of the W-31-109-ENG-38. under contract U.S. Government retains a Accordingly, the U.S. Government retains a nonexclusive, royalty-free license to publish or reproduce the publisted form of this contribution, or allow others to do so, for U.S. Government purposes.
Figure 1: Prototype Beam Measurement System Schematic. 


\section{DISCLAIMER}

Portions of this document may be illegible in electronic image products. Images are produced from the best available original document. 


$$
\mathrm{V}_{\mathrm{T}}=\frac{2 \mathrm{i}_{\text {ave }} \ell \mathrm{Q}_{\mathrm{o}}}{\mathrm{C}_{\mathrm{T}} \beta \mathrm{c}} \cdot \frac{\sin (\mathrm{N} \pi \mathrm{fT})}{(\mathrm{N} \pi \mathrm{fT})} \cdot \frac{\sin \left(\frac{\mathrm{N} \pi \mathrm{f} \ell}{\beta \mathrm{c}}\right)}{\left(\frac{\mathrm{N} \pi \mathrm{f} \ell}{\beta \mathrm{c}}\right)}
$$

where:
$\mathrm{V}_{\mathrm{T}}=$ peak induced signal in volts

$\mathrm{i}_{\mathrm{ave}}=$ average bunched beam current

$\ell=$ pickup electrode length in meters

$\mathrm{C}_{\mathrm{T}}=$ total capacitance in farads

$\beta \mathrm{c}=$ ion beam velocity $\mathrm{m} / \mathrm{s}$

$\mathrm{Q}_{0}=$ lightly loaded $\mathrm{Q}$ of system

$\mathrm{N}=$ bunching harmonic number

$\mathrm{T}=$ bunch width in sec.

$\mathrm{f}=$ bunch repetition rate in $\mathrm{Hz}$
$(4.26 \mathrm{~V})$

(10.0 microamperes)

$(0.040 \mathrm{M})$

$\left(48.5 \times 10^{-12} \mathrm{~F}\right)$

$\left(2.55 \times 10^{6} \mathrm{~m} / \mathrm{s}\right)$

(1)

$\left(10^{-9} \mathrm{sec}\right)$

$\left(1.2125 \times 10^{7} \mathrm{~Hz}\right)$
Equation 1 was solved for the ion velocity, bunching, and harmonic number stated. Physical constants such as resonating capacitance and $Q$ value were determined by laboratory measurement. Pickup electrode length " $\ell$ " was chosen on the basis of available space, beam sensitivity, ion beam velocity, and acceptable response over several bunching harmonics.

\section{BEAM SIGNAL PROCESSING}

As shown in Eq. 1, the peak value of signal strength " $\mathrm{V}_{\mathrm{T}}$ " is equal to 0.42 volts per microampere of bunched beam. We do not critically couple beam induced power out of the resonant electrode, instead, a high input impedance $\mathrm{RF}$ buffer amplifier is used to sense voltage " $\mathrm{V}_{\mathrm{T}}$ ". Amplifier $\mathrm{A}_{1}$ can be connected to sense all of the beam induced voltage across $L_{T}$, or can be tapped at lower voltage points to accommodate higher beam intensity. Unity gain amplifier $A_{1}$ has an input impedance of 1.5 megohms in parallel with 2.0 picofarads, which does not significantly load the electrode's shunt impedance of 0.19 megohm.

Processing the $12 \mathrm{MHz}$ beam signal into a DC voltage requires a two-step procedure to achieve acceptable linearity and dynamic range. Consider the introduction of a high-level RF signal slightly shifted in frequency from the beam. The summed offset and beam signals can then be diode detected to obtain amplitude and phase information. We, in fact, generate an accurate, stable offset signal which is removed from the fundamental frequency of bunching by $1250 \mathrm{~Hz}$. This offset signal is coupled to the beam pick-up electrode and processed by the same circuitry used for beam induced signals. Offset and bunching signals sum together and appear as a lowfrequency envelope modulation at the RF detector. This process is represented by the characteristics of two sine waves of slightly different frequency, as their vectors sum through $2 \pi$ radians of phase.

$$
f(t)=A \sin (\omega t) \pm B \sin (K \omega t)
$$

where:

$A=$ amplitude of the beam induced signal

$B=$ amplitude of offset oscillator drive

$\mathrm{t}=$ time

$\mathrm{K}=$ offset frequency factor $=1.0001031$

$\omega=2 \pi \mathrm{f}(\mathrm{f}=12.125 \mathrm{MHz})$

At $100 \%$ modulation $(\mathrm{A}=\mathrm{B})$ the $1250 \mathrm{~Hz}$ modulation envelope will take on the characteristics of a sine squared function.

If we limit the percentage of modulation to $25 \%$ or less, the distortion effect becomes negligible and a clean $1250 \mathrm{~Hz}$ sine wave results. System parameters are set such that the highest accelerated beam currents result in no more than $25 \%$ envelope modulation at the RF detector.

Beam tests were carried out to verify the accuracy of Eq. 1. As maximum sensitivity is desired for this test, we configured buffer amplifier $A_{1}$ to sense the total voltage across inductor $\mathrm{L}_{\mathrm{T}}$. Since maximum beam current should not produce more than $25 \%$ modulation of RF offset voltage, the magnitude of $\mathrm{V}_{\mathrm{T}}$ is set by what the buffer amplifiers can handle. This limited our test beam to a maximum of 1.0 microampere. Figure 2a shows the RF offset voltage level across inductor $\mathrm{L}_{\mathrm{T}}$. Notice that, under zero beam conditions, the peak amplitude of $\mathrm{V}_{\mathrm{T}}$ is equal to 4.0 microamperes of beam current. If 1.0 microamperes of beam is present, the peak value of $\mathrm{V}_{\mathrm{T}}$ is equal to 4.0 microamperes plus or minus 1.0 microampere, as shown in Fig. 2b. Detector diode D1 recovers the modulation envelope of $1250 \mathrm{~Hz}$. Active bandpass filter number one (ABFI), shown in Fig. 1, has a $Q$ of ten and unity gain. This $Q$ value sets the system bandwidth at $125 \mathrm{~Hz}$ for beam intensity measurements. The amplitude of the $1250 \mathrm{~Hz}$ sine wave is calibrated at this point by adjusting

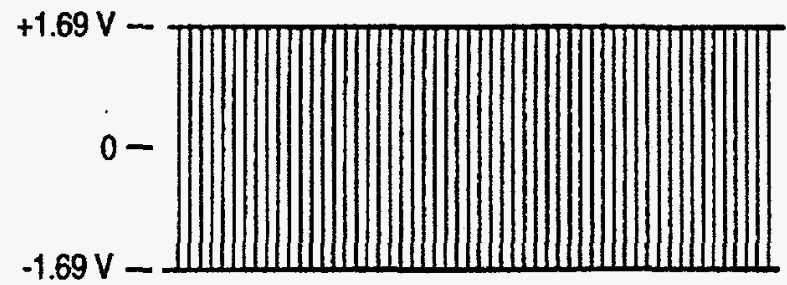

Fig. 2(a)

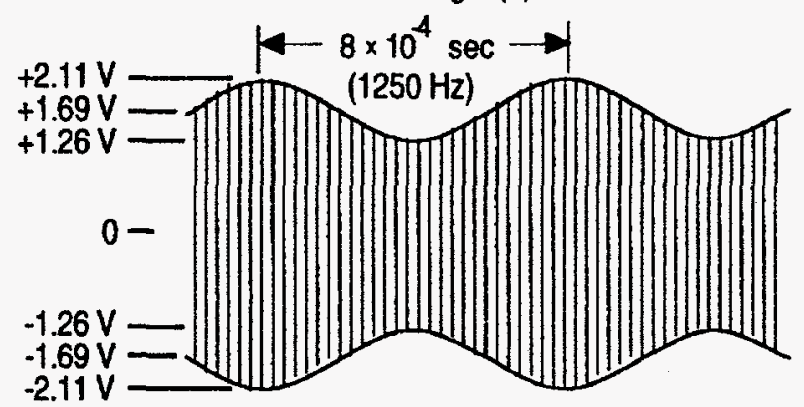

Fig. 2(b)

Figure 2: RF offset voltage level diagram. 
the gain of low noise amplifier $A_{5}$. Noise voltage amplitude is one millivolt peak to peak at point " $\mathrm{C}$ " in Fig. 1 , which is equivalent to 50 picoamperes of beam. For 1.0 microampere beams, the signal amplitude at point " $\mathrm{C}$ " rises to 20 volts peak to peak. The full wave active rectifier features $1 \%$ linearity over the beam intensity range of 1.0 microamperes to 500 picoamperes.

\section{BEAM PHASE MEASUREMENT}

Figure 1 shows the beam phase channel consists of two diode limiters, two active band pass filters, and a phase locked loop (PLL). Care was taken to use temperature stable components for the active bandpass filters and PLL voltage controlled oscillator (VCO) circuit. We have used the system for measuring injection phase at the Positive Ion Injector and results have been excellent. We have plans to install two more measuring stations at ATLAS. Phase measurements of accelerated beam exhibit less than $0.5^{\circ}$ of jitter over a beam current range of 1.0 microampere to 1.0 nanoamperes. The phase locked loop provides a constant phase signal because its internal VCO is very stable. The free running PLL VCO frequency is always within one hertz of $1250 \mathrm{~Hz}$, therefore the phase error during a millisecond beam drop out is small.

\section{SUMMARY}

The beam monitor described in this paper is useful for measuring the phase and magnitude of nanoampere ion beams. We plan to improve the sensitivity and noise performance by raising electrode $Q$ and reducing system bandwidth. A future goal is to develop a low cost beam monitor which accurately measures the current and phase of picoampere ion beams. Sensitive, low noise instrumentation will be needed if radioactive beams are developed at ATLAS.

*This work was supported by the U.S. Department of Energy, Nuclear Physics Division, under contract W-31-109-ENG-38.

\section{REFERENCES}

[1] R.C. Pardo, L.M. Bollinger, and K.W. Shepard, Nucl. Instrum. and Methods B24/25, 746 (1987).

[2] L.M. Bollinger, et al., Nucl. Instrum. and Methods B79, 753 (1993).

[3] S. Takeuchi and K.W. Shepard, "A Sensitive BeamBunch Phase Detector," Nucl. Instrum. and Methods 227, 217-219 (1984).

[4] A.D. Frawley and J.D. Fox, "A Spiral-Resonator Beam Phase Detector," Nucl. Instrum. and Methods 204, 37. 40 (1982). 